\title{
On the Link between Sensorimotor Adaptation and Sensory Recalibration
}

\author{
Fabrice R. Sarlegna ${ }^{1}$ and Pierre-Michel Bernier ${ }^{2}$ \\ ${ }^{1}$ Institute of Movement Sciences, Centre National de la Recherche Scientifique and Aix-Marseille Université, 13009 Marseille, France, and ${ }^{2}$ Brain Imaging \\ Center, Department of Psychology, University of California-Santa Barbara, Santa Barbara, California 93106 \\ Review of Ostry et al.
}

Our environment is constantly changing, as is the state of our own musculoskeletal system. This imposes a permanent adaptive pressure on the sensorimotor system, which must be updated across development and aging to allow smooth interactions with the world. Consider the case of having to reach an apple when a $1 \mathrm{~kg}$ load is suddenly added on the arm. Such experimental manipulation mimics, on a much shorter time scale, a change in limb dynamical properties that could result from too much feasting during the holidays. Typically, the sudden addition of a load causes the hand movement to fall short of the targeted object. A solution to this problem is to adaptively increase the force exerted on the arm to displace it further. The CNS's capacity to adaptively tailor the motor output to the current conditions is thought to be achieved through prolonged exposure, taking into account the successful actions as well as the committed errors.

Consistent with the idea that sensorimotor adaptation requires a change in motor output, neurophysiological work

Received June 14, 2010; revised July 13, 2010; accepted July 15, 2010.

This work was supported by funds from the French government (DGCIS: Research and Development Program) and from the Région PACA to F.R.S. and the Fyssen Foundation to P.-M.B. We thank the reviewers for their helpful comments.

Correspondence should be addressed to Dr. Fabrice Sarlegna, Institute of Movement Sciences, Centre National de la Recherche Scientifique and Aix-Marseille Université, 163 Avenue de Luminy, 13009 Marseille, France. E-mail: fabrice.sarlegna@univmed.fr.

DOI:10.1523/JNEUROSCI.3040-10.2010

Copyright $\odot 2010$ the authors $\quad 0270-6474 / 10 / 3011555-03 \$ 15.00 / 0$ in nonhuman primates has demonstrated that when facing novel dynamical environments, changes in motor cortical areas restore behavioral efficiency (Gandolfo et al., 2000). However, surprisingly little is known about whether sensorimotor adaptation is also associated with changes in sensory systems. In particular, does one's somatosensory estimate of limb position also change during sensorimotor adaptation?

Recently in The Journal of Neuroscience, Ostry et al. (2010) addressed this issue by using a classic force-field adaptation paradigm. Subjects had to reach to a visual target while holding the handle of a robot. During the movement, the robot generated velocity-dependent forces orthogonal to the direction of the reach, causing subjects' arm movements to substantially deviate from a straight line. Consistent with previous work, subjects adapted their movement pattern to the requirements of the new dynamic environment, as indicated by the recovery of a relatively straight hand trajectory [Ostry et al. (2010), their Figs. $1 A$ and $6 A$ ].

The original feature of the study by Ostry et al. (2010) was to assess subjects' perception of hand position before and after adaptation to the force field. In these perceptual tests, subjects again had to reach toward a visual target, but as soon as the movement started, visual representations of the limb movement (i.e., a cursor) and the target position were removed. The robot then pushed the hand either to the left or to the right, and subjects had to verbally report whether they felt their arm deviate in either direction. The magnitude and direction of the force pulse was adjusted according to an iterative algorithm to precisely determine the perceptual boundary between left and right.

The main result of Ostry et al. (2010) was that after force-field adaptation, subjects' perception of hand position was shifted in a direction consistent with the force applied during adaptation to counteract the force field [Ostry et al. (2010), their Fig. $2 \mathrm{~A}$ ]. For example, after subjects had become adapted to a force pushing their arm to the left and thus exerted more force to the right, their perception of hand position was shifted to the right. Because hand position could only be assessed via somatosensory feedback coming from muscles, joints, and skin, the authors concluded that motor adaptation was associated with somatosensory recalibration. These findings were confirmed and extended in a second experiment using passive perceptual tests.

These results are important because they constitute the first account of a somatosensory recalibration following forcefield adaptation. For several decades, sensory recalibration has been associated with adaptation to visuomotor perturbations such as displacing prisms and cursor shifts (Harris, 1963; Simani et al., 2007; Cressman and Henriques, 2010). In these cases, the alteration of hand visual feedback produces a conflict between visual 
and somatosensory afferent signals (Fig. $1 A$ ), prompting the recalibration of one or both sensory modalities to resolve the conflict (Harris, 1963; Simani et al., 2007; Cressman and Henriques, 2010). However, a novel force field differs in a fundamental way from a prismatic deviation or a visuomotor rotation: at no point does it introduce a conflict between visual and somatosensory cues (Fig. $1 B$ ). It thus remains an open question what functional mechanism leads to somatosensory recalibration during force-field adaptation, let alone which brain areas underlie this reorganization. Here we briefly examine these issues and formulate some suggestions.

Current models of sensorimotor control suggest that the brain continuously uses an efference copy of motor commands to predict the sensory consequences of actions (Desmurget and Grafton, 2000; Flanagan et al., 2003; Shadmehr et al., 2010). Upon introduction of the novel force field, an error arises between the predicted sensory consequences (for instance a straight-ahead movement) and the actual reafferent sensory signals (i.e., a laterally deviated movement) (see Fig. 1B). Flanagan et al. (2003) showed that adaptation to a novel force field involves the iterative updating of the predictor (i.e., the forward model). In this light, one of the key results of Ostry et al. (2010) is that somatosensory recalibration was only observed when subjects actively reached to the target within the novel force field, but not when they had their limb passively moved by the robot along the same (deviated) kinematics as during active reaching. This suggests that somatosensory recalibration is tightly linked to the adaptive process and does not merely arise from sensory experience. The lack of intersensory conflict during force-field adaptation thus raises an intriguing possibility: the adaptive process may have similarly shifted all sensory modalities that contribute to the estimation of limb position. Hence, in parallel to the change in somatosensory perception observed by Ostry et al. (2010), adaptation may have also caused a shift in subjects' visually perceived limb position. Such multimodal sensory recalibration would appear necessary to maintain a coherent percept of hand position in the absence of a visuoproprioceptive conflict. To our knowledge, no work has yet addressed the recalibration of the visual estimate of limb position following force-field adaptation. However, it should be noted that Brown et al. (2007) previously showed that force-
A

Visuomotor rotation

B New force field

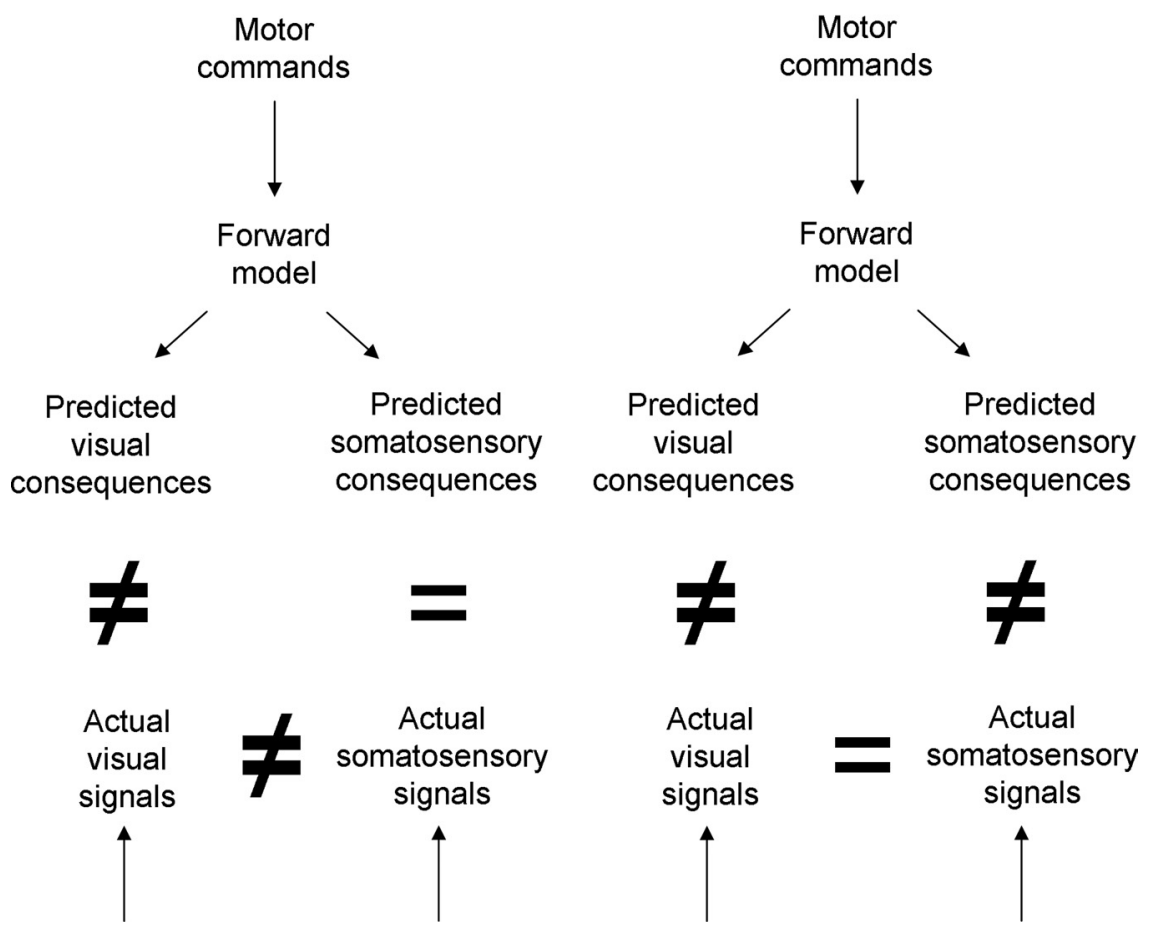

Figure 1. It is thought that a forward model can predict the sensory consequences of motor commands (Desmurget and Grafton, 2000; Flanagan et al., 2003; Shadmehr et al., 2010). A, When visual feedback is artificially altered, such as during visuomotor rotation, a conflict arises between the predicted and actual visual consequences of the movement (depicted by " $\neq{ }^{\prime \prime}$ ), but not between the predicted and actual proprioceptive consequences of the movement (depicted by " $="$ "). The conflict between visual and somatosensory estimates prompts the recalibration of these modalities (Harris, 1963; Simani et al., 2007; Cressman and Henriques, 2010). $\boldsymbol{B}$, In the case of a novel force field, the visual and proprioceptive consequences of the movement are affected similarly, and there is no conflict between the two sensory estimates. It is thus possible that in addition to the somatosensory shift observed by 0stry et al. (2010), a similar visual shift occurred to maintain a homogeneous percept of hand position.

field adaptation influences the visual estimate of target motion.

Evidence for a perceptual shift in both visual and somatosensory modalities may provide insight into potential structures mediating the adaptive change in the sense of limb position. Specifically, it would point to the involvement of higherorder associative regions, such as the superior parietal lobule, which is known to integrate visual, somatosensory, and efferent signals from motor areas to provide an integrated estimate of the state of the body (Kalaska, 1996; Desmurget and Grafton, 2000; Graziano et al., 2000). Hence, in addition to plastically altering low-level sensory maps in primary sensory and motor areas, as proposed by Ostry et al. (2010), force-field adaptation may modify the way these maps are read out by associative parietal regions. Modifying perception in higher-level areas may afford flexibility, perhaps accounting for the observation of Ostry et al. (2010) that subjects still presented a reliable bias in their somatosensory judgments when asked to come back into the laboratory $24 \mathrm{~h}$ after force-field adaptation. While this finding speaks to the robustness of the recalibration, it is unlikely that subjects had a shifted somatosensory estimate of limb position as they went about their normal activities in the intervening day, since this would have given rise to motor inaccuracies (and led to deadaptation). Hence, analogous to our capacity to retrieve specific sensorimotor mappings for different mechanical contexts (e.g., reaching accurately with bare hands or with heavy gloves), sensory representations may be differently "interpreted" according to context, giving rise to distinct limb state estimates.

In conclusion, the evidence for somatosensory plasticity provided by Ostry et al. (2010) extends current knowledge regarding adaptive reorganization in the human brain when facing novel dynamical environments. It indicates that forcefield adaptation affects sensory systems as well as motor systems. Hopefully, future 
work will shed light on the neurophysiological underpinnings of this sensory recalibration, providing insight into its functional role.

\section{References}

Brown LE, Wilson ET, Goodale MA, Gribble PL (2007) Motor force field learning influences visual processing of target motion. J Neurosci 27:9975-9983.

Cressman EK, Henriques DY (2010) Reach adaptation and proprioceptive recalibration following exposure to misaligned sensory input. J Neurophysiol 103:1888-1895.
Desmurget M, Grafton S (2000) Forward modeling allows feedback control for fast reaching movements. Trends Cogn Sci 4:423-431.

Flanagan JR, Vetter P, Johansson RS, Wolpert DM (2003) Prediction precedes control in motor learning. Curr Biol 13:146-150.

Gandolfo F, Li C, Benda BJ, Schioppa CP, Bizzi E (2000) Cortical correlates of learning in monkeys adapting to a new dynamical environment. Proc Natl Acad Sci US A 97: 2259-2263.

Graziano MS, Cooke DF, Taylor CS (2000) Coding the location of the arm by sight. Science 290:1782-1786

Harris CS (1963) Adaptation to displaced vi- sion: visual, motor or proprioceptive change? Science 140:812-813.

Kalaska JF (1996) Parietal cortex area 5 and visuomotor behavior. Can J Physiol Pharmacol 74:483-498.

Ostry DJ, Darainy M, Mattar AA, Wong J, Gribble PL (2010) Somatosensory plasticity and motor learning. J Neurosci 30:5384-5393.

Shadmehr R, Smith MA, Krakauer JW (2010) Error correction, sensory prediction, and adaptation in motor control. Annu Rev Neurosci 33:89-108.

Simani MC, McGuire LM, Sabes PN (2007) Visual-shift adaptation is composed of separable sensory and task-dependent effects. J Neurophysiol 98:2827-2841. 